\title{
Influence of zinc oxide nanorods on an orientationally ordered fluid comprising soft-bent dimers
}

\author{
PRAGNYA SATAPATHY, SRIVIDHYA PARTHASARATHI, D S SHANKAR RAO, \\ MADHUBABU KANAKALA, C V YELAMAGGAD and S KRISHNA PRASAD* D \\ Centre for Nano and Soft Matter Sciences, Bengaluru 560013, India \\ *Author for correspondence (skprasad@cens.res.in)
}

MS received 26 May 2018; accepted 26 June 2018; published online 21 August 2018

\begin{abstract}
Bent-core liquid crystals (LCs) introduced a whole new dimension to the science of LCs. Besides re-emphasizing the importance of the shape of the molecule, they brought in phases with symmetry, not known earlier. Another sub-class of systems that is emerging is that of soft-bent molecules. In contrast with the bent-core molecules, here the bend is achieved through the parity of the aliphatic linker that connects two monomers. They hold the unique advantage that a simple variation of temperature can favour different conformer states and thus govern the self-assembled structure. A highlight of the power of this route is seen in terms of the discovery of a new type of nematic, viz., twist-bend nematic. Investigations on not only this phase but also the regular nematic that often precedes it have received significant attention in the last few years. Here we present results on the regular nematic phase of a binary mixture comprising such a soft-bent dimer, known in the literature as CB7CB, by incorporating zinc oxide nanorods (NRs) into the system. The NRs with an aspect ratio of $\sim 7.2$, not very different from that of LCs, further accentuate the importance of shape and shape anisotropy of the entities. Specifically, we observe that the nematic-isotropic transition temperature increases by $\sim 1.9 \mathrm{~K}$ even for a low concentration of $4 \%$ NRs. While the dielectric anisotropy decreases, birefringence shows a substantial increase, adding to the complexity of the influence. Upon addition of minute amount of NRs (1\%), while the splay elastic constant gets enhanced, its bend counterpart not only gets reduced but retains the convex-shaped thermal profile seen for the parent mixture.
\end{abstract}

Keywords. Nano-soft composite; liquid crystal; soft-bent dimer; nanorods; zinc oxide.

\section{Introduction}

Chirality in achiral systems is a well-studied phenomenon in condensed matter systems, for example, $\alpha$-quartz, $\mathrm{NaClO}_{3}$ molecules arranged in helical order, sulphur, selenium, etc. Generation of chiral structures in achiral molecules has attracted much interest and has been well achieved in liquid crystals (LCs), especially in the form of bent-core substances. The recently discovered twist-bend nematic $\left(\mathrm{N}_{\mathrm{TB}}\right)$ phase provides a new perspective to these efforts [1-5]. The present article describes the influence of zinc oxide nanorods (NRs) on the behaviour of the nematic LC phase in a material that exhibits the $\mathrm{N}_{\text {Tв }}$ phase at a lower temperature. Although there are reports of the influence of zinc oxide nanoparticles on LC behaviour [6-11], to our knowledge, there are none on NRs of this material.

Keeping the broad readership in mind we provide in the following, a brief description of the relevant liquid-crystalline phases in this article and their elastic behaviour [12]. All these phases exist between the isotropic liquid (Iso, figure 1a) and the crystal $(\mathrm{Cr}$, figure $1 \mathrm{e})$. The simplest of the liquidcrystalline phases is the nematic $(\mathrm{N})$, which can be termed simply as an orientationally ordered fluid (figure 1b). This phase is characterized by a preferred orientation direction for the molecules labelled the director $\mathbf{n}$ and no positional order. When the molecules are chiral, the director precesses in space, giving rise to a helix as seen in the cholesteric $\left(\mathrm{N}^{*}\right)$ phase (figure 1c); the local director is perpendicular to the helical axis $\left(\theta_{0}=\pi / 2\right)$. Till recently, $N^{*}$ was the only helical counterpart of the $\mathrm{N}$ phase. However, recently, a theoretical prediction [1] and series of experiments [13-17] subsequently have generated much interest in a new helical phase, termed the twist-bend nematic $\left(\mathrm{N}_{\mathrm{TB}}\right)$ phase. This feature stresses the true soft matter character of the phase, wherein subtle changes either to the molecule or to the environment can bring about large impact on the phase structure. In the $\mathrm{N}_{\mathrm{TB}}$ phase the director forms an angle $0<\theta_{0}<\pi / 2$ with the helical axis, the pitch itself having nanometre length scales (figure 1d). Although the phase has been reported in bent-core compounds, the $\mathrm{N}_{\mathrm{TB}}$ phase exhibited by dimeric molecules has been well characterized [13-17].

The origin of the $\mathrm{N}_{\mathrm{TB}}$ can be appreciated by looking at the elasticity of the nematic medium. The nematic ordering arises due to the long and highly polarizable molecules, which try to align parallel to each other, the structure being stabilized by van der Walls dispersion forces. The orientational order in nematic is described in terms of the ability of the molecules to be oriented along a particular direction, referred to as the 


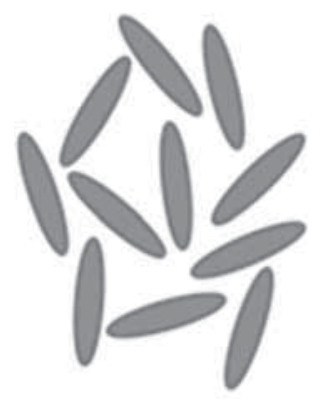

(a)

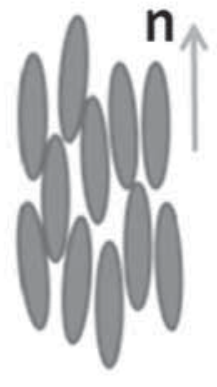

(b)

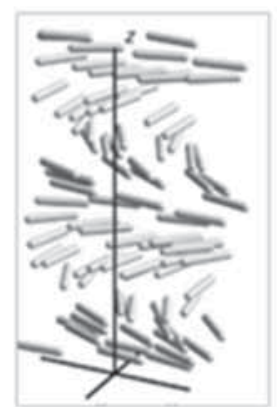

(c)

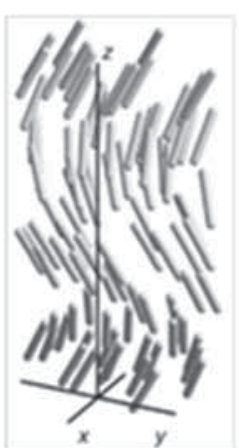

(d)

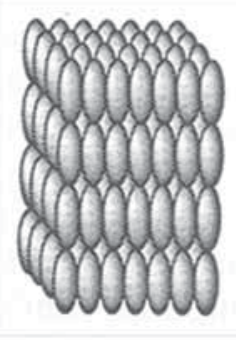

(e)

Figure 1. Schematic representation of the molecular arrangement in the (a) isotropic, (b) nematic, (c) chiral nematic, (d) twist-bend nematic and (e) crystal phases. In (b), $\mathbf{n}$ represents the average orientation direction of the liquid crystal molecules.

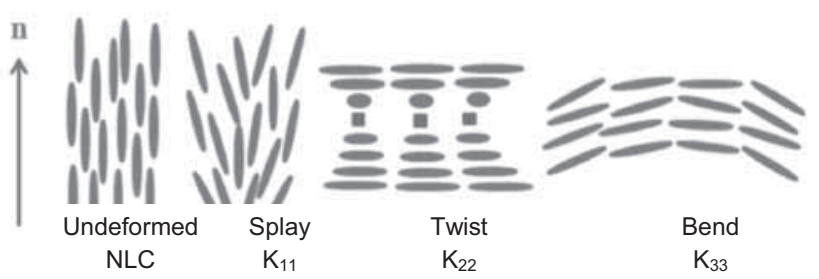

Figure 2. Principal elastic deformations in a nematic liquid crystal.

director (n) and quantified in terms of the order parameter $S$. In macroscopic samples, non-uniform director distributions can easily occur owing to different boundary conditions or external fields. Three principal elastic deformations, viz., splay, twist and bend, can describe the non-uniform distributions (see figure 2). The associated elastic energy density due to the director curvature can be written as

$$
\begin{aligned}
f_{\mathrm{N}}= & \frac{1}{2}\left[K_{11}(\nabla \bullet \hat{n})^{2}+K_{22}(\hat{n} \bullet \nabla \times \hat{n})^{2}\right. \\
& \left.+K_{33}(\hat{n} \times \nabla \times \hat{n})^{2}\right] .
\end{aligned}
$$

The terms on the RHS of equation (1) represent, respectively, the splay, twist and bend terms with $K_{11}, K_{22}$ and $K_{33}$ as the corresponding elastic constants. For the $\mathrm{N}$ phase, $K_{11}-K_{33}$ are positive, which ensures that the ground state has uniform director distribution. Dozov [1] proposed that in the nematic phase of bent molecules, $K_{33}$ can become negative. In this case, the ground state should not be uniform but will have a local spontaneous bend, leading to an equilibrium twist-bend structure as a possible state, and the phase is referred to as the twist-bend nematic $\left(\mathrm{N}_{\mathrm{TB}}\right)$. Its structure is schematically shown in figure 1d. Experiments have established the occurrence of the twist-bend state, although negative values of $K_{33}$ have not been observed. A molecular necessity for realizing the $\mathrm{N}_{\mathrm{TB}}$ phase appears to be the bent shape of the entity. We have recently demonstrated [15] that in binary systems comprising a soft-bent dimer giving rise to the $\mathrm{N}_{\mathrm{TB}}$ phase, a significant amount of loading of a rod-like compound still sustains the twist-bend structure. The importance of the aspect ratio (length/diameter ratio) in stabilizing the liquid crystallanity of the material needs no emphasis. Since the aspect ratio of the added rod component in Ref. [15] is $\sim 4$, a typical value for LC molecules, we were curious to see the influence of the added component having a much higher aspect ratio. To achieve this we have incorporated NRs of zinc oxide that present an aspect ratio of $\sim 7.2$. Of course, unlike the LC molecules, the $\mathrm{ZnO}$ NRs are far stiffer. Here we present early results of the thermal, dielectric and elastic constant investigations, in which we concentrate only on the influence in the ordinary nematic phase.

\section{Experimental}

For these investigations, we have chosen a binary system that we have characterized earlier [15]. It comprises the dimer $\alpha$, $\omega$ bis(4, 4'-cyanobiphenyl)heptane CB7CB for short) with a structurally similar monomer, the well-known n-heptyloxy cyanobiphenyl (7OCB). The molecular structures of both these compounds along with the phase sequence exhibited by them are given in figure 3 . To be noted is the chemical nature of the dimer: The two rigid cyanobiphenyl arms are connected through a 'soft' aliphatic spacer having an odd parity ( 7 carbon atoms). It has been established that the parity of this spacer has an important role to play in deciding the mesomorphic properties. Also to be noted is that while 7OCB is a rod-like molecule, $\mathrm{CB} 7 \mathrm{CB}$ has a bent character arising from the odd parity of the spacer. However, unlike molecules belonging to the bent-core class of materials, the bend in the present case is achieved through the much softer, and thus easily amenable, aliphatic chain. Therefore we refer to CB7CB as a 'soft-bent' molecule. We shall revisit these points later. The structural similarity between the two components helps in an excellent homogenization of the mixture. Besides, the $\mathrm{N}_{\text {TB }}$ phase 
survives even with a higher loading factor of rod-like nematic, an attractive feature, but out of scope for the present article. $\mathrm{CB} 7 \mathrm{CB}$ was synthesized in our laboratory while $7 \mathrm{OCB}$ was procured from commercial sources (Aldrich) and used as is. The dimer shows $\mathrm{N}$ and $\mathrm{N}_{\text {TB }}$ phases below the isotropic (Iso) liquid, whereas 7OCB has only the $\mathrm{N}$ mesophase. The host LC mixture (HLC) used for all the experiments reported here consisted of $64 \mathrm{~mol} \%$ of $7 \mathrm{OCB}$ in CB7CB. It presents the $\mathrm{N}$ phase over a thermal range of $50 \mathrm{~K}$, extending down to $29^{\circ} \mathrm{C}$ and the $\mathrm{N}_{\mathrm{TB}}$ phase below it.

Vertically grown $\mathrm{ZnO}$ NRs were synthesized by the hydrothermal synthesis route. This involved seed-mediated

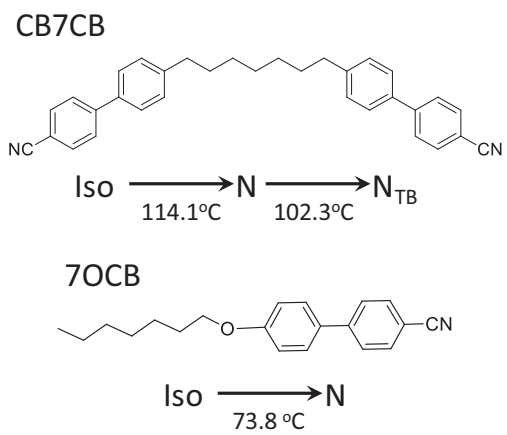

Figure 3. Molecular structures and transition temperatures for the $\mathrm{N}_{\mathrm{TB}}$ material CB7CB and the rod-like nematic 7OCB, which constitute the host HLC mixture. growth where the pre-deposited seeds help the nucleation and growth of preferentially vertical NRs [18], and helps in obtaining NRs of desired aspect ratios [19]. Seeds were prepared in the aqueous solution of equimolar $20 \mathrm{mM}$ concentration of zinc nitrate hexahydrate $\left(\mathrm{Zn}\left(\mathrm{NO}_{3}\right)_{2} \cdot 6 \mathrm{H}_{2} \mathrm{O}\right)$ and hexamethylene tetramine (purchased from Alfa Aesar) of each of $100 \mathrm{ml}$ and stirring at $80^{\circ} \mathrm{C}$ for $10 \mathrm{~min}$; after this the solution was drop cast on clean glass plates. The films containing the seeds were immersed vertically in the same solution used for the seed preparation and then fluxed in a hot air oven at $90^{\circ} \mathrm{C}$ for $5 \mathrm{~h}$. After this the films were taken out and washed with DI water to remove the particles attached. The successful vertical growth of $\mathrm{ZnO}$ NRs has been confirmed through the scanning electron microscopy (SEM). The FESEM images of these $\mathrm{ZnO}$ NRs, obtained using a MIRA 3 LHU model (Tescan), are shown in figure 4a. To disperse the NRs in LCs, the NRs were cleaved from the glass films and dispersed in methanol. The dispersed solution was drop casted on a silicon substrate and checked using SEM (figure 4b). The NRs exhibit a very-well-defined rod shape with a hexagonal cross-section; the size histograms (figure 4c and d) indicate the diameter and length to be $259 \pm 4 \mathrm{~nm}$ and $1.86 \pm 0.048 \mu \mathrm{m}$, respectively, providing an aspect ratio of $7.2 \pm 0.05$.

Experiments have been performed on the composites containing $1 \%$ and $4 \%$ of $\mathrm{ZnO} \mathrm{NRs}$; for brevity, they are referred to as HLC1Z and HLC4Z, respectively. For the electrical studies, samples were sandwiched between indium tin oxide (ITO) coated glass plates procured from a
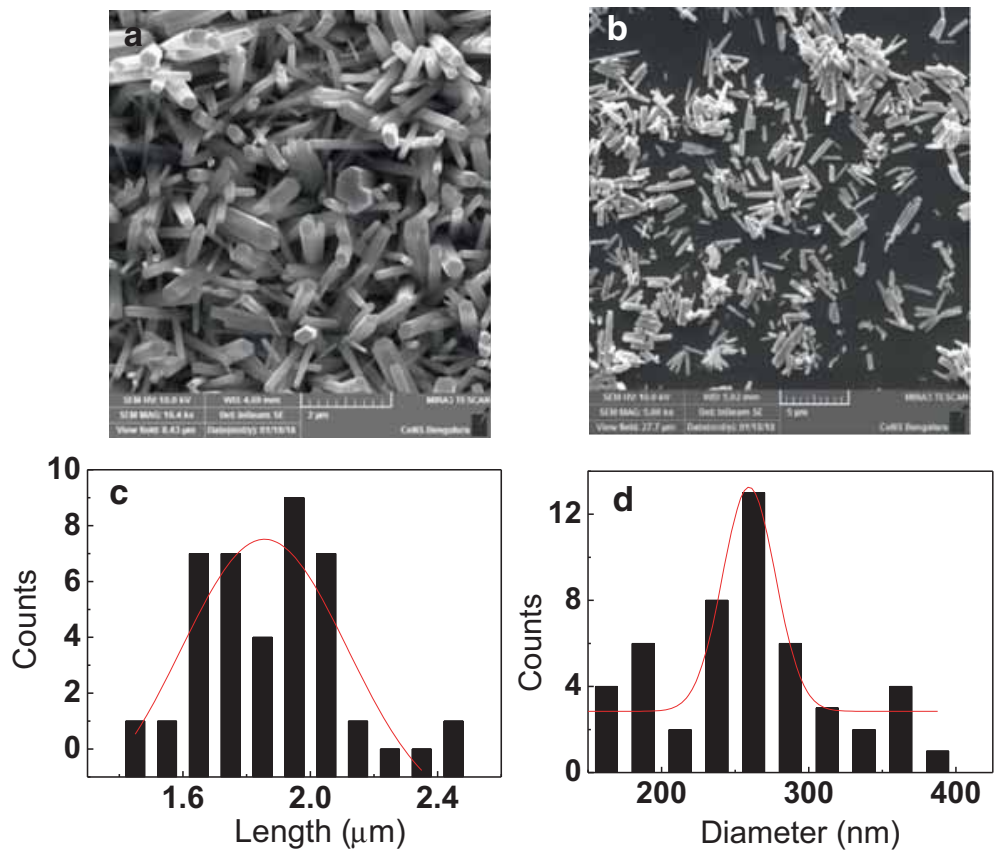

Figure 4. SEM images of $\mathrm{ZnO} \mathrm{NRs}$ (a) grown on glass substrate and (b) drop casted on a substrate after dispersing in methanol; (c, d) size histograms, from which the length and diameter of the NRs are estimated to be $1.86 \pm 0.048 \mu \mathrm{m}$ and $259 \pm 4 \mathrm{~nm}$, respectively. 
commercial source (AWAT, Poland) with the cell gap of $\sim 7.7 \mu \mathrm{m}$. The inner surfaces of the substrates have a polymer layer to promote a unidirectional planar alignment of the LC molecules. Permittivity and elastic constant measurements were performed at a constant frequency of $10 \mathrm{kHz}$ using a high precision LCR meter (Agilent 4284A) capable of applying an oscillating voltage up to $20 \mathrm{~V}$. The procedure, based on the Deuling analysis [20,21], was implemented by a userwritten routine scripted in MATLAB [22,23].

\section{Results}

\subsection{Calorimetry}

Figure 5 shows the DSC profiles obtained at a rate of $5^{\circ} \mathrm{C} \min ^{-1}$ in the cooling mode in the vicinity of the Iso-N transition for HLC and the two composites. For all the three materials clear and sharp thermal peaks are seen, indicating that the mixtures are quite homogeneous with no observable phase segregation between LC and the NRs. This is further corroborated by polarizing optical microscopy observations. The most important feature to be noticed for the composites is the positive shift in $T_{\mathrm{NI}}$, the nematic-isotropic transition temperature, taken here to be the peak point of the profiles. A substantial increase is seen by adding the NRs: the enhancement $\Delta T=1.2 \mathrm{~K}$ for HLC1Z and $1.9 \mathrm{~K}$ for HLC4Z. As already stated, the NRs have a larger aspect ratio than the LC molecules. Thus the observation here is in line with the expectation that an increase in the aspect ratio of the constituents increases $T_{\mathrm{NI}}$. It should, however, be noted that the concentration of NRs is quite low. Thus it can be argued that the stiffness of the ZnO NRs is quite effective in asserting and even enhancing the shape-anisotropy-related stability of the LC phase. Investigations have been performed with materials chemically similar to the rod-like entity (7OCB) employed in the present studies, as the host into which nanoparticles are doped. To our knowledge, such studies have not employed $\mathrm{ZnO}$ NRs. Therefore we consider here, for comparison, measurements in which carbon nanotubes [24]

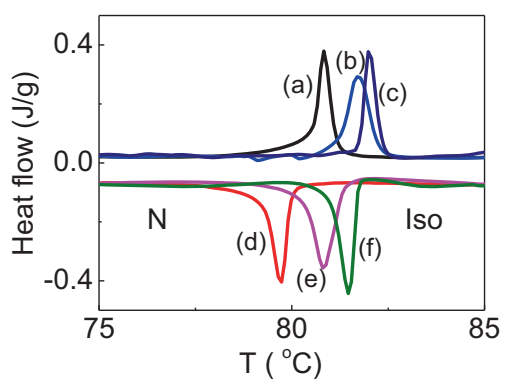

Figure 5. Differential scanning calorimetry profiles in the vicinity of the nematic-isotropic transition in the (a)-(c) heating and (d)-(f) cooling modes for (a, d) HLC, (b, e) HLC1Z and (c, f) HLC4Z. To be specifically noted is the increase in the transition temperature upon addition of NRs. with a generally much larger aspect ratio are employed. With hardly an exception, induction of the anisotropic nanoparticle results in a reduction of $T_{\mathrm{NI}}$. For example, Basu et al [25] found that $T_{\mathrm{NI}}$ reduces by $\sim 1 \mathrm{~K}$. In contrast, it is observed here that not only is the $T_{\mathrm{NI}}$ increased but also the magnitude of increase is also large. For the latter, an explanation could at least be that the concentration of $\mathrm{ZnO}$ NRs used here is much higher $(4 \%)$ than those using CNT $(<1 \%)$, the limitation being caused by the inability to satisfactorily disperse CNT in LCs at high loads. With this as the background we made a mixture of HLC with CNT (single-walled CNT, with a nominal purity of $>90 \%$, procured from Heji, Hongkong, and having dimensions of $\sim 2 \mathrm{~nm}$ diameter and $\sim 500 \mathrm{~nm}$ length). Surprisingly, we observed that the $0.05 \%$ CNT doped into HLC results in an increase in the transition temperature by $\sim 2 \mathrm{~K}$ ! Perhaps the much larger aspect ratio of CNT $(\sim 250)$ enables it to bring about the same effect at much lower concentrations than the $\mathrm{ZnO} \mathrm{NRs}$ with a smaller $(\sim 7.2)$ aspect ratio. In the light of these observations, we speculate that the presence of the soft-bent component (CB7CB) is responsible for the trend reversal in $T_{\mathrm{NI}}$. More experiments are needed to conclude on these features.

\subsection{Thermal variation of permittivity}

Permittivity measurements were performed in two geometries, wherein the director in the $\mathrm{N}$ phase is parallel $\left(\varepsilon_{\|}\right)$or perpendicular $\left(\varepsilon_{\perp}\right)$ to the probing field. Whereas for the surface-determined $\perp$ geometry, the measuring voltage was $0.5 \mathrm{~V}$, to get the $\|$ alignment a larger voltage $(20 \mathrm{~V})$ was used; in both cases, the frequency of the field was $10 \mathrm{kHz}$. Figure 6 shows the variation of $\varepsilon_{\|}$and $\varepsilon_{\perp}$ as a function of temperature in the isotropic and nematic phases for the HLC and for the nanocomposites. Qualitatively all three materials behave quite similarly, with $\varepsilon_{\|}\left(\varepsilon_{\perp}\right)$ increasing (decreasing) as the temperature is lowered from the Iso to the $\mathrm{N}$ phase. Notable differences do exist, though. The first feature observed is that for the composites there is an upward shift in the transition temperature, by magnitudes comparable to those observed in the calorimetric data discussed earlier. More importantly, the permittivity values are influenced by the presence of NRs. For HLC1Z, $\varepsilon_{\|}$is hardly changed, but $\varepsilon_{\perp}$ is reduced

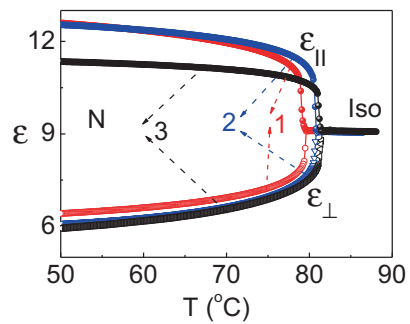

Figure 6. Thermal variation of permittivity parallel $\left(\varepsilon_{\|}\right)$and perpendicular $\left(\varepsilon_{\perp}\right)$ to the nematic director normalized with respect to the isotropic value ( $\left.\varepsilon_{\text {Iso }}\right)$ for the HLC material (dataset 1) and for the nanocomposites, HLC1Z (dataset 2) and HLC4Z (dataset 3). 


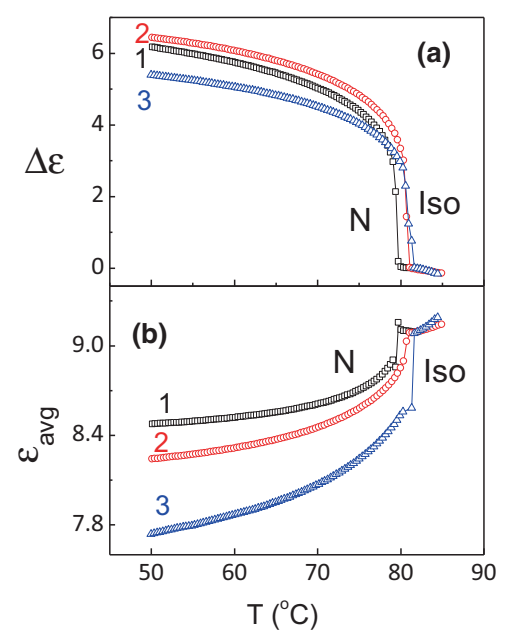

Figure 7. Thermal variation of permittivity (a) anisotropy and (b) average for HLC (1) and the nanocomposites, HLC1Z (2) and HLC4Z (3).

by $4 \%$. When the NR concentration increases (HLC4Z), $\varepsilon_{\|}$ also gets affected, diminishing by $9 \%$, and $\varepsilon_{\perp}$ by $5.5 \%$. The difference between the two permittivity values yields the dielectric anisotropy $\left(\Delta \varepsilon=\varepsilon_{\|}-\varepsilon_{\perp}\right)$. The thermal variation of this anisotropy is shown in figure 7a for HLC and the two composites. For the $1 \%$ composite, there is a slight increase in $\Delta \varepsilon$, but the $4 \%$ composite exhibits a substantial decrease, largely due to the reduction in $\varepsilon_{\|}$. The dielectric anisotropy can be taken to be a measure of the orientational order parameter. This is especially true for strongly polar compounds such as those constituting the HLC mixture. Thus, the behaviour of $\Delta \varepsilon$ would indicate a lowering of the order parameter for HLC4Z composite. However, a reduced order parameter should have lowered the nematic-isotropic transition temperature also, contrary to the observations. Another anisotropic parameter that is also considered to be a measure of the order parameter is the birefringence of the medium. The birefringence values, measured at a temperature of $T_{\text {red }}=$ $T_{\mathrm{NI}}-T=25 \mathrm{~K}$ using a tilting compensator, are $0.2,0.25$ and 0.3 for HLC, HLC1Z and HLC4Z materials, respectively, clearly pointing to an increase in the order parameter. Thus, we suggest that the presence of NRs favours a better dipolar coupling between the host molecules, and thus an improved cancellation of dipole moment contribution along the director direction. A clear indication of such an antiparallel coupling known in alkyl cyanobiphenyls is parametrized in terms of the average permittivity $\varepsilon_{\mathrm{avg}}=\left(\varepsilon_{\|}+2 \varepsilon_{\perp}\right) / 3$. To be very specific, in the absence of any coupling, the value of $\varepsilon_{\text {avg }}$ at any temperature in the nematic should be comparable to that extrapolated from the isotropic value; the presence of coupling is indicated by the value being lower than that extrapolated. Figure $7 \mathrm{~b}$ demonstrates that the coupling is prominent in the pure HLC as well as the nanocomposites, and that the effect is stronger with the addition of the NRs. Further investigations are on to get a better understanding of this feature.

\subsection{Frank elastic constants}

We used the well-known electric-field-induced Freedericksz transformation technique to measure the splay and bend elastic constants for HLC and the HLC1Z composite. Let us recall the essential features of this method. Initially, the molecules are aligned parallel to the substrate surface. Given the positive dielectric anisotropy of the system, application of a voltage greater than a certain threshold voltage $\left(V_{\text {th }}\right)$ normal to the substrate plane will reorient the molecules perpendicular to the substrate. The sample capacitance was used to detect and measure this change. Using $V_{\text {th }}$ and $\Delta \varepsilon$, the splay $\left(K_{11}\right)$ and bend $\left(K_{33}\right)$ elastic constants are calculated using the Deuling analysis [20-23]. The actual expressions employed are

$$
\begin{aligned}
& K_{11}=\varepsilon_{0} \Delta \varepsilon V_{\mathrm{th}}^{2} / \pi^{2}, \\
& \frac{C(V)-C_{\perp}}{C_{\perp}}=\gamma-\frac{2 \gamma}{\pi} \frac{V_{\mathrm{th}}}{V} \sqrt{1+\gamma \sin ^{2} \phi_{\mathrm{m}}} \int_{0}^{\phi_{\mathrm{m}}} \\
& \qquad\left\{\frac{\left(1+\kappa \sin ^{2} \phi\right)\left(1-\sin ^{2} \phi\right)}{\left(1+\gamma \sin ^{2} \phi\right)\left(\sin ^{2} \phi_{\mathrm{m}}-\sin ^{2} \phi\right)}\right\}^{1 / 2} \cos \phi d \phi .
\end{aligned}
$$

Here, $\varepsilon_{0}$ is the permittivity of free space, $\kappa=\left(K_{33} / K_{11}\right)-1$, $\gamma=\left(\varepsilon_{\|} / \varepsilon_{\perp}\right)-1$ and $\phi_{\mathrm{m}}$ is the angle between the director and the walls of the substrate with $\phi_{\mathrm{m}}$ being the value in the midplane of the sample.

The value of $K_{11}$ can be directly obtained through equation (2) with the help of experimentally determined $V_{\text {th }}$ and $\Delta \varepsilon$ values, whereas extraction of $K_{33}$ requires a full profile fitting to equation (3), carried out with the help of a MATLAB script $[22,23]$. The profiles of capacitance $v$. applied voltage $V$ obtained for HLC and the HLC1Z composite at several temperatures are shown in figure $8 \mathrm{a}$ and $\mathrm{b}$, respectively. In all the cases the threshold is observed to be quite sharp across the entire $\mathrm{N}$ range. As expected, $V_{\text {th }}$ shows a monotonic increase when the temperature is lowered. Interestingly, the profiles taken at a fixed reduced temperature $\left(T_{\text {red }}=\right.$ $15 \mathrm{~K}$ ) bring out clearly that the threshold voltage increases when the NRs are added (see figure 8c). This is not surprising since the dopant is a stiffer rod than the host LC molecules.

Figure 9 presents the thermal dependence of the splay elastic constant for HLC and the nanocomposite. For both materials, $K_{11}$ exhibits a monotonic variation with temperature, a standard feature seen for rod-like bent core as well as the soft-bent compounds [12-17,22,23,26,27]. However, addition of $\mathrm{ZnO}$ NRs increases the magnitude of $K_{11}$ by as much as $46 \%$ at the lowest temperature measured. In contrast with the monotonic behaviour of $K_{11}$, the bend elastic constant presents a convex-shaped thermal profile (CSTP). Figure 10 shows that the CSTP, a feature known for HLC, is not only retained for the composite but enhanced in features as well. Firstly, the point at which there is a trend reversal in $K_{33}$ occurs much closer to the isotropic temperature. Secondly, at any $T_{\text {red }}$, the composite has a lower $K_{33}$ than the parent 

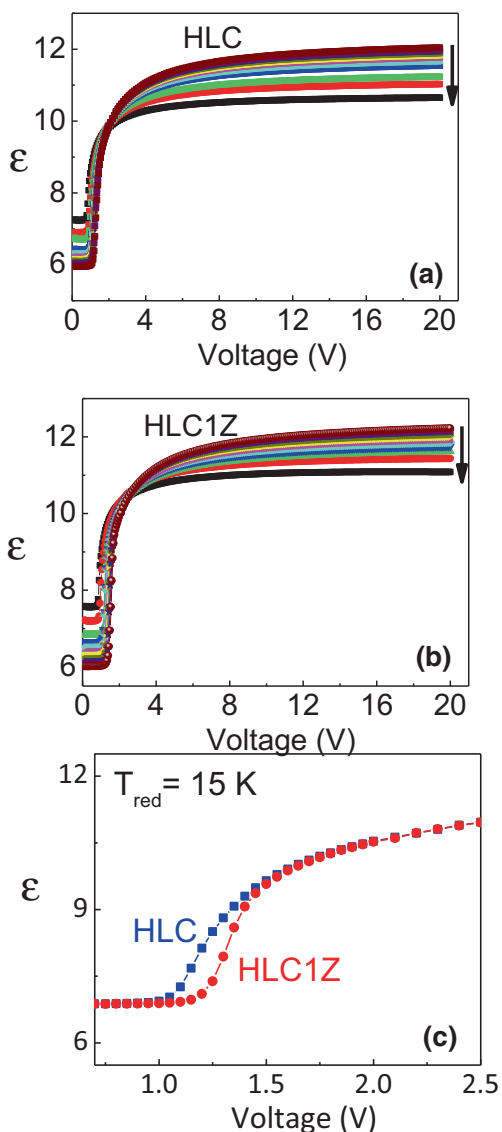

Figure 8. Freedericksz transition profiles at various reduced temperatures $\left(T_{\mathrm{red}}=T_{\mathrm{NI}}-T\right)$ in the (a) HLC and (b) HLC1Z materials. The $T_{\text {red }}$ values for the different sets are, from the top, $1,3, \ldots, 30 \mathrm{~K}$ at the interval of 3 . In each case the knee point of the rising curve gives an estimate of the threshold voltage. Enlarged view of the profiles at a fixed reduced temperature of $T_{\text {red }}=15 \mathrm{~K}$ clearly shows that the threshold voltage increases when the NRs are added.

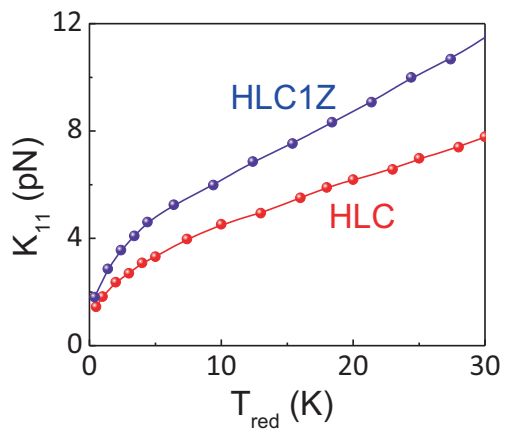

Figure 9. Thermal variation of the Frank splay elastic constant for HLC and the HLC1Z nanocomposite.

LC (HLC). Based on the fact that with hardly any exception, the CSTP behaviour is not observed in pure compounds but only in binary mixtures (see Ref. [23] and references therein) with a rod-like component, we have argued [14] that CSTP

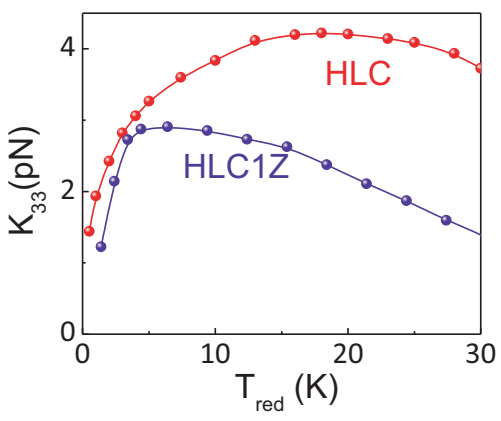

Figure 10. Thermal variation of the Frank bend elastic constant for HLC and the HLC1Z nanocomposite.

in bent-core calamitic systems arises due to the frustration in the packing of the two differently shaped molecules. The incorporation of $\mathrm{ZnO}$ NRs accentuating this feature lends credence to this argument. Detailed experiments with the higher concentration of $\mathrm{ZnO}$ NRs will throw more light on this.

\section{Conclusion}

In summary, we have investigated thermal, dielectric and elastic properties of a host LC system consisting of a soft-bent component and a calamitic one, in its pure form and upon addition of $\mathrm{ZnO}$ NRs. The NRs bring about substantial changes in all these properties and show the power of tunability of the associated parameters through this route. The aspect ratio of the NRs and its concentration are parameters that we are now investigating to obtain a multidimensional understanding of such nano-soft composites. Photoluminescence, an inherent property of $\mathrm{ZnO}$, is also being probed to understand its anisotropic character. This may result in (i) a better understanding of the influence of hard NRs in controlling/tuning the LC character of the system, and (ii) realizing electrically switchable self-assembled photoluminescent materials. Work will also be extended to the underlying twist-bend nematic phase.

\section{Acknowledgements}

This research work was supported by Thematic project (SR/NM/TP-25/2016), Nano Mission, Department of Science and Technology (DST), New Delhi, India.

\section{References}

[1] Dozov I 2001 Europhys. Lett. 56247

[2] Panov V P, Nagaraj M, Vij J K, Panarin Y P, Kohlmeier A, Tamba M G et al 2010 Phys. Rev. Lett. 105167801

[3] Cestari M, Frezza E, Ferrarini A and Luckhurst G R $2011 \mathrm{~J}$. Mater. Chem. 2112303 
[4] Chen D, Porada J H, Hooper J B, Klittnick A, Shen Y M, Tuchband M et al 2013 Proc. Natl. Acad. Sci. 11015931

[5] Henderson P A and Imrie C T 2011 Liq. Cryst. 381407

[6] Martínez-Mirandaa L J, Traister K M, Meléndez-Rodríguez I and Salamanca-Riba L 2010 Appl. Phys. Lett. 97223301

[7] Joshi T, Kumar A, Prakash J and Biradar A M 2010 Appl. Phys. Lett. 96253109

[8] Yuan K, Li F, Chen L, Li Y and Chen Y 2012 J. Phys. Chem. C 1166332

[9] Pandey K K, Misra A K and Manohar R 2016 Appl. Nanosci. 6141

[10] Zheng Z, Butynska R, Serrano C V, Marty J, Mingotaud C and Kahn M L 2016 Chem. Eur. J. 2215614

[11] Ma Q, Ma S and HuangY M 2018 Mater. Lett. 218237

[12] Chandrasekhar S 1994 Liquid crystals (Cambridge: Cambridge University Press)

[13] Cestari M, Berart S D, Dunmur D A, Ferrarini A, de la Fuente M R, Jackson D J B et al 2011 Phys. Rev. E 84031704

[14] Parthasarathi S, Rao D S S, Palakurthy N B, Yelamaggad C V and Krishna Prasad S 2017 J. Phys. Chem. B 121896

[15] Parthasarathi S, Rao D S S, Palakurthy N B, Yelamaggad C V and Krishna Prasad S 2016 J. Phys. Chem. B 1205056
[16] Krishnamurthy K S, Palakurthy N B and Yelamaggad C V 2017 J. Phys. Chem. B 1215447

[17] Yun C J, Vengatesan M R, Vij J K and Song J K 2015 Appl. Phys. Lett. 106173102

[18] Pal A K and Mohan D B 2015 Appl. Surf. Sci. 333244

[19] Sun H, Luo M, Weng W, Cheng K, Du P, Shen G et al 2008 Nanotechnology 1939560

[20] Deuling H J 1972 Mol. Cryst. Liq. Cryst. 19123

[21] Uchida T and Takahashi Y 1981 Mol. Cryst. Liq. Cryst. 72 133

[22] Madhuri P L, Krishna Prasad S, Hiremath U S and Yelamaggad C V 2014 Appl. Phys. Lett. 104241111

[23] Parthasarathi S, Rao D S S, Fodor C K and Krishna Prasad S 2014 J. Phys. Chem. B 11814526

[24] Yadav S P and Singh S 2016 Prog. Mater. Sci. 8038

[25] Basu R, Sigdel K P and Iannacchione G S 2011 Eur. Phys. J. E 3434

[26] Sathyanarayana P, Mathew M, Li Q, Sastry V S S, Kundu B, Le K V et al 2010 Phys. Rev. E 81010702

[27] Wiant D, Gleeson J T, Eber N, Fodor-Csorba K, Jakli A and Toth-Katona T 2005 Phys. Rev. E 72041712 